\title{
Effects of Resveratrol on Inflammatory Bowel Disease: A Review
}

\author{
Elizabeth R. Abouaf-Tabet, Fred W. Kolkhorst, and Mee Young Hong* \\ School of Exercise and Nutritional Sciences, San Diego State University, San Diego, CA 92182-7251,USA
}

Received: March 10, 2014; Accepted: June 14, 2014; Published: June 18, 2014

*Corresponding author: Mee Young Hong, Department of Exercise and Nutritional Sciences, San Diego State University, 5500 Campanile Drive, San Diego CA 92182-7251,USA, Tel.: (619) 594-2392; Fax.: (619) 594-6553; E-mail address: mhong2@mail.sdsu.edu

\begin{abstract}
Inflammatory bowel disease (IBD) is an autoimmune disease characterized by chronic inflammation in the colon and small intestine. IBD produces many symptoms that can cause discomfort and a modified lifestyle. IBD has no cure, only drugs used to suppress its inflammation, which have exhibited harmful side effects. Resveratrol, 3,5,40 -trihydroxy-trans-stilbene, is a natural phenol with anti-inflammatory attributes. Studies have found consistent results showing that resveratrol supplementation in experimental rodent models of IBD can reduce inflammatory biomarkers. This review presents experimental animal models of IBD showing that resveratrol supplementation can down-regulate inflammatory pathways of MAPK and NF- kb, lessen COX-2, modify cytokines, diminish leukocytes, alter intestinal microflora, and decrease clinical symptoms in vivo, all of which contribute to an improved state of the disease. These outcomes, however, have not yet been studied in naturally occurring IBD in humans. Future research should attempt and refine to determine if resveratrol could be an effective therapy for IBD in humans.
\end{abstract}

Keywords: Inflammatory bowel disease; Ulcerative colitis; Crohn's disease; Inflammation; Resveratrol

\section{Abbreviations}

BI: Bowel Injected; CD: Crohn's Disease; CI: Colon Injected; COX: Cyclooxygenase; DSS: Dextran Sodium Sulfate; FOXP3: Forkhead Box P3; GSH: Glutathione; IBD: Inflammatory Bowel Disease; ICAM-1: Intracellular Adhesion Molecule 1; IFN- $\gamma$ : Interferon Gamma; IL: Interleukin; INOS: Inducible Nitric Oxide; LPO: Lipid Peroxide; MAPK: Mitogen Activated Protein Kinase; MDA: Malondialdehyde; MIG: Monokine Induced by Interferon Gamma; MPO: Myeloperoxidase; NF-кb: Nuclear Factor Kappa B; NO: Nitric Oxide; O.I: Orally Injected; OXA: Oxazolone; PGE: Prostaglandin E; PGES: Prostaglandin E Synthase; PG-PS: Peptidoglycan Polysaccharide; RANTES: Regulated on Activation Normal T cell Expressed and Secreted; RI: Rectum Injected; ROS: Reactive Oxygen Species; SAA: Serum Amolyoid A; SIRT: Silent Mating Type Information Regulation; SOD: Superoxide Dismustase; SPHK1: Sphingosine Kinase 1; STAT: Signal Transducer and Activator of Transcription; TGF: Transforming Growth Factor; TNBS: 2,4,6-Trinitrobenzenesulfonic acid; TNF:
Tumor Necrosis Factor; UC: Ulcerative Colitis; VCAM- 1: Vascular Cell Adhesion Molecule.

\section{Introduction}

The Center for Disease Control and Prevention (CDC) estimates 1.4 million diagnosed cases of inflammatory bowel disease (IBD) in the United States costing the health care industry between $\$ 1.7$ and $\$ 5.2$ billion each year [1,2]. IBD accounts for more than 700,000 physician visits, 100,000 hospitalizations, and disability in 119,000 patients annually in the United States [1]. IBD, which includes Ulcerative Colitis (UC) and Crohn's disease (CD), is an autoimmune disease and poses pained digestion and malnutrition risk due to a compromised gastrointestinal tract. The etiology of this disease remains unknown, but is thought to be a combination of immune responses, environmental factors and genetic dynamics [3]. Although IBD alone is not fatal, those with UC and CD have a six times increased risk of developing colon cancer [4]. Current drugs and surgeries used to treat the symptoms of IBD can cause negative side effects [1,5]. For these reasons, it is important to find a treatment that is able to reduce the development of IBD with little side effects.

Resveratrol, 3,5,40 -trihydroxy-trans-stilbene, is a natural phenol and phytoalexin that can be found in red wine, grapes, peanuts, and Japanese knotweed. It has been widely researched for its antioxidant properties, most specifically in cancer and cardiovascular diseases [6-9]. In some human studies, resveratrol can decrease signs of inflammation with virtually no side effects $[8,10]$. No human studies, however, exist to show anti-inflammatory effects of resveratrol in IBD. The objective of this review is to discuss the effects of resveratrol on inflammatory signaling pathways, inflammatory biomarkers, intestinal microflora and clinical symptoms in animal models of experimental IBD. To our knowledge, this is the first review assessing the effect of resveratrol on IBD.

\section{Resveratrol's Effect on Experimental-IBD \\ Inhibited signaling pathways}

Resveratrol reduces inflammation through mitogen activated protein kinase (MAPK) and nuclear factor kappa B (NF-кB) 
pathways. P38 MAPK, one of four, general MAPK signaling systems in mammals, has been recognized as a critical part in the pathogenesis of CD $[11,12]$. Activation of p38 MAPK, by the proinflammatory cytokine tumor necrosis factor (TNF)- $\alpha$, is down regulated with resveratrol administration $[11,13]$.

Reactive oxygen species (ROS), bacteria, and inflammatory cytokines activate NF- $\kappa B$, [14] the pathway specifically linkedto the pathogenesis of UC $[12,15,16]$. With resveratrol supplementation, NF- $\mathrm{B}$ p 65 subunit activity is inhibited through attenuating attenuate nuclear factor of kappa B inhibitor, alpha

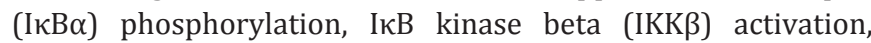
extracellular signal-regulated kinases (ERK) phosphorylation, signal transducer and activator of transcription 3 (STAT3) activation and NF- $\mathrm{BB}$ DNA binding in animal models [16-18]. Resveratrol also amplifies silent mating type information regulation 1 (SIRT1) gene expression, [18,19] which indirectly inhibitsNF- $\mathrm{kB}$.

\section{Reduced oxidative stress}

Oxidative stress increases inflammation by stimulating MAPK and NF- $\mathrm{B}$ pathways. Resveratrol supplementation, however, decreases several biomarkers of ROS including malondialdehyde (MDA), p22 $2^{\text {phox }}$ and gp91 $1^{\text {phox }}$, and myeloperoxidase (MPO) $[15,20,21-25]$. In one study using experimental colitis, specifically induced by 2,4,6-trinitrobenzenesulfonic acid(TNBS), glutathione (GSH)was reduced by $44 \%$ [21]. Even a moderately low dose of resveratrol $(10 \mathrm{mg} / \mathrm{kg} /$ day) increased GSH $[20,21]$ and superoxide dismutase (SOD) [20].

\section{Reduced proinflammatory mediators}

Excess nitric oxide (NO) has been associated with the etiology and progression of IBD [26,27]. Inducible nitric oxide synthase (iNOS), which initiates NO production, is up regulated in a state of chronic intestinal inflammation [27]. Resveratrol generally diminishes iNOS protein expression $[13,16,28]$ and NO production $[29,30]$. One study dissimilarly found resveratrol to raise NO levels above baseline values [21]. Since constitutive NO is also involved in gut homeostasis, [27] restoring NO after resveratrol supplementation may be beneficial for gastrointestinal maintenance of equilibrium, however, prolonged large quantity of NO would be harmful.

Cyclooxygenase (COX) is an enzyme present in sites of inflammation [31,32]. With dextran sodium sulfate (DSS) treatment, COX-2 potentially increases 2.3 -fold [30] which can be attenuated by Resveratrol $[13,17,18,24,25,28]$ both directly and through suppressed ROS levels [31]. The effects of resveratrol supplementation on COX-1 have been equivocal; one study showed no significant changes in COX-1[25] while others showed COX-1 was decreased [18,24].

\section{Diminished leukocytes}

Neutrophils increase in experimental IBD [28]. Resveratrol administration significantly reduces markers for neutrophil infiltration including MPO activity, intracellular adhesion molecule 1 (ICAM-1) and vascular cell adhesion molecule 1
(VCAM-1) [15,20,21-25,28,33]. In one study, the resveratroltreated group had $20 \%$ less neutrophils in the lamina propria compared to the placebo group [28].

IBD comprises excess $\mathrm{CD}^{3+}$ and $\mathrm{CD}^{4+} \mathrm{T}$-cells; [34] however, as a result of resveratrol they are decreased to normal levels $[18,23,28]$. Forkhead box P3 cells are typically reduced in IBD, [35] but increase with Resveratrol [23] signifying decreased natural T regulatory cells and reduced severity of IBD [36]. CD11 $\mathrm{b}^{+}$and Gr$1^{+}$were increased during resveratrol supplementation leading to decreased effector $\mathrm{T}$-cell function and a decline in clinical symptoms [33].

\section{Modulated cytokines}

TNF- $\alpha$, the central, most effective cytokine in IBD, becomes less pronounced with resveratrol in experimental animal models of IBD $[13,15,18,20,28,33,37,38]$. Reduced TNF- $\alpha$ can also directly decline VCAM-1 and ICAM-1, [39] which further improves IBD symptoms by inhibiting neutrophil function. Other inflammatory cytokines similarly decrease with resveratrol administration including interleukin (IL)-1 $1 \beta,[13,15,18,24,33,38]$ IL-6,[13,15,18,23,33,38] IL-8[20] and IL-12 [33]. IFN- $\gamma$, which is elevated in DSS-included colitis $[28,40]$ also reduces with resveratrol supplementation [18,20,23]. Anti-inflammatory cytokine IL-10 increases with Resveratrol [13,23] which further prevents activation of the NF- $\kappa$ B pathway. Additionally, chemokines including monokine induced by interferon gamma (MIG) (CXCL9), macrophage inflammatory protein-1 gamma (MIP-1 $\gamma$ ) (CCL9), monocyte chemotactic protein-1 (MCP-1) (CCL2), CXCL10 ${ }^{+}, \mathrm{CXCR}^{+}$and RANTES (regulated on activation, normal $\mathrm{T}$ cell expressed, secreted, CCL5) decreased in animal models of IBD supplementing Resveratrol [23,33,41].

\section{Modification of intestinal microflora}

Rise in colonic permeability in IBD allows interaction between microbiota and immune responses [30,42]. Resveratrol administration reduces Escherichia coli; Enterococci, and total bacteria load in the intestine 1.0 - 3.0 orders of magnitude $[23,30,37]$. Resveratrol simultaneously increases Lactobacilli and Bifidobacteria 1.0 order of magnitude, peaking after 20 days of administration [23,30]. Resveratrol is not only able to reduce bacteria overgrowth, but also limit bacteria translocation into sub-epithelial tissues, [23] which can be a crucial factor in disease progression.

\section{Reduced clinical symptoms}

Clinical symptoms of IBD include weight loss, abdominal pain, fever and bloody diarrhea [5]. In a majority of studies, weight loss was less in animals given Resveratrol [13,15,16,20,21,23, $24,28,33,39]$. While few studies showed no difference between groups $[37,38]$. Complete recovery in body weight was only seen at a resveratrol dose of $100 \mathrm{mg} / \mathrm{kg} /$ day [18]. Colon weight, $[13,21,24,25]$, histological scores,[13,15,18,20,21-25,30,33] fibrosis,[38] rectal bleeding and diarrhea,[13,15,20,22] also diminished after resveratrol supplementation, signifying reduced inflammation. Studies were inconclusive regarding colon length and its relation to inflammation $[13,16,18,23-25,28,30]$. 
Table 1: Studies Examining the Effect of Resveratrol on Experimental IBD.

\begin{tabular}{|c|c|c|c|c|c|c|}
\hline Author, Year & $\begin{array}{l}\text { Animal Models of } \\
\text { IBD }\end{array}$ & $\begin{array}{l}\text { Route } \\
\text { \&Timing of } \\
\text { Model }\end{array}$ & Dose & $\begin{array}{l}\text { Duration of } \\
\text { Resveratrol }\end{array}$ & Physical/Clinical Outcomes & Other Measured Outcomes \\
\hline $\begin{array}{l}\text { Abdallah et al., } \\
\text { 2011[21] }\end{array}$ & $\begin{array}{l}\text { OXA in Wister } \\
\text { albino rats }\end{array}$ & ri., Day 1 & 10 & 7 days & $\begin{array}{l}\downarrow \text { Ulcerative area } \\
\downarrow \text { Wt. loss } \\
\downarrow \text { Colon wt. } \\
\downarrow \text { Histological score }\end{array}$ & $\begin{array}{l}\downarrow \text { MPO activity } \\
\downarrow \text { MDA activity } \\
\downarrow \text { CAM- } 1^{\text {a }} \\
\downarrow \text { VCAM-1 } \\
\downarrow \text { aPO } \\
\downarrow \mathrm{NO}^{\mathrm{a}} \\
\downarrow \mathrm{GSH}^{\mathrm{a}}\end{array}$ \\
\hline $\begin{array}{l}\text { Abdin et al., } \\
2013[22]\end{array}$ & $\begin{array}{l}100 \text { T. gondii cysts } \\
\text { in C57BL/10 mice }\end{array}$ & c.i., Day 1 & 10 & 14 days & $\begin{array}{l}\downarrow \text { Rectal bleeding } \\
\downarrow \text { Diarrhea } \\
\downarrow \text { Histological score }\end{array}$ & $\begin{array}{l}\downarrow \text { MPO activityc } \\
\downarrow \text { SphK1 activity } \\
\nexists \text { Capase-3 activity }\end{array}$ \\
\hline $\begin{array}{l}\text { Bereswill et al., } \\
2010[23]\end{array}$ & $\begin{array}{l}\text { DSS in male and } \\
\text { female C57BL/ } 6 \\
\text { mice }\end{array}$ & o.i., Day 3 & 10 & 10 days & $\begin{array}{l}\downarrow \text { Wt. loss } \\
\downarrow \text { Bacteria translocation } \\
\downarrow \text { Total Bacteria Load } \\
\downarrow \text { Enterococcic }^{\mathrm{a}} \\
\downarrow \text { E. Colic } \\
\downarrow \text { Lactobacilli/ Bifidobacteria }^{\mathrm{a}} \\
\downarrow \text { Lt. of small intestines }\end{array}$ & $\begin{array}{l}\downarrow \text { IL- } 6^{\mathrm{a}} \\
\downarrow \text { MPO-7+a } \\
\downarrow \text { MCP- }{ }^{\mathrm{a}} \\
\downarrow \text { IFN- } \gamma^{\mathrm{a}} \\
\uparrow \mathrm{FOXP3^{+a }} \\
\downarrow \mathrm{CD}^{+\mathrm{a}} \\
\uparrow \mathrm{Ki}^{+} 67+^{\mathrm{b}} \\
\uparrow \mathrm{IL}-10^{\mathrm{a}}\end{array}$ \\
\hline $\begin{array}{l}\text { Cui et al., } \\
2010[28]\end{array}$ & $\begin{array}{l}\text { DSS in male and } \\
\text { female C57BL/6J } \\
\text { mice }\end{array}$ & o.i., First 7 days & 42 & 70 days & $\begin{array}{l}\downarrow \text { Wt. loss } \\
\uparrow \text { Colon lt. }\end{array}$ & $\begin{array}{l}\downarrow \text { CD3 }^{\text {+a }} \\
\downarrow \text { Neutrophils } \\
\downarrow \text { COX-2 } \\
\downarrow \text { CNF- } \\
\downarrow \text { TN } \\
\downarrow \text { iNOS } \\
\downarrow \text { p53 } \\
\downarrow \text { p }\end{array}$ \\
\hline $\begin{array}{l}\text { Larrosa et al., } \\
2010[41]\end{array}$ & $\begin{array}{l}\text { DSS in male Fischer } \\
\text { F344rats }\end{array}$ & o.i., Last 8 days & 2.1 & 29 days & $\begin{array}{l}\downarrow \text { E. Coli }^{\mathrm{a}} \\
\downarrow \text { Enterobacteria }^{\mathrm{a}} \\
\nexists \text { Enterococci } \\
\nexists \text { Lactobacilli } \\
\nexists \text { Bifidobacteria } \\
\nexists \text { Clostridia } \\
\nexists \text { Wt. loss } \\
\nexists \text { Colon wt. \& lt. }\end{array}$ & 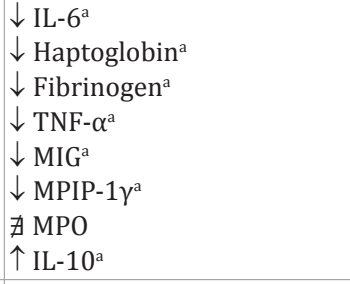 \\
\hline $\begin{array}{l}\text { Larrosa et al., } \\
2009[30]\end{array}$ & $\begin{array}{l}\text { TNBS in male } \\
\text { Winstar rats }\end{array}$ & o.i., Last 5 days & 1 & 25 days & 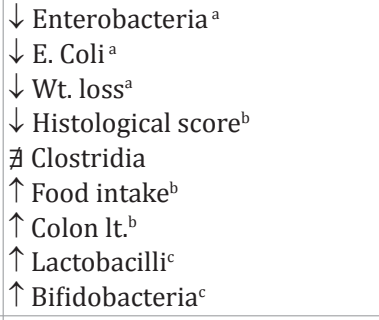 & $\begin{array}{l}\downarrow \text { PGE }_{2}{ }^{\mathrm{b}} \\
\downarrow \mathrm{COX}^{\mathrm{b}} \mathrm{2}^{\mathrm{b}} \\
\downarrow \mathrm{NO}^{\mathrm{b}} \\
\downarrow \mathrm{PTGES}^{\mathrm{b}} \\
\nexists \text { Haptoglobin } \\
\nexists \text { Albumin }\end{array}$ \\
\hline $\begin{array}{l}\text { Martin et al., } \\
2006[25]\end{array}$ & $\begin{array}{l}\text { TNBS in male } \\
\text { Winstar rats }\end{array}$ & c.i., Day1 & 10 & 14 days & $\begin{array}{l}\downarrow \text { Macroscopic damage score } \\
\downarrow \text { Colon lt. \& wt. }\end{array}$ & $\begin{array}{l}\downarrow \text { MPO activity } \\
\downarrow \text { TNF- } \alpha^{\mathrm{a}} \\
\downarrow \text { NF- } \mathrm{B} \text { p } 65^{\mathrm{b}} \\
\downarrow \mathrm{COX}-2^{\mathrm{b}} \\
\nexists \mathrm{COX}-1 \\
\nexists \mathrm{PGD}_{2} \\
\uparrow \mathrm{PGE}_{2}^{\mathrm{c}}\end{array}$ \\
\hline $\begin{array}{l}\text { Martin et al., } \\
2004[24]\end{array}$ & $\begin{array}{l}\text { PG-PS in female } \\
\text { Lewis rats }\end{array}$ & c.i., Day 3 & 10 & 4 days & $\begin{array}{l}\downarrow \text { Macroscopic damage score }{ }^{a} \\
\downarrow \text { Wt. loss } \\
\downarrow \text { Colon lt. \& wt. } \\
\downarrow \text { COX-2 in mucosa }\end{array}$ & $\begin{array}{l}\downarrow \mathrm{IL}^{2}-1 \beta^{\mathrm{c}} \\
\downarrow \mathrm{PGD}_{2}{ }^{\mathrm{a}} \\
\downarrow \mathrm{MPO}^{\mathrm{a}} \\
\nexists \mathrm{PGE}_{2}\end{array}$ \\
\hline $\begin{array}{l}\text { Rahal et al., } \\
2012[38]\end{array}$ & $\begin{array}{l}\text { DSS in female } \\
\text { C57BL/ } 6 \text { mice }\end{array}$ & b.i., Day 1 & 100 & 27 days & $\begin{array}{l}\downarrow \text { Histologic fibrosis score } \\
\nexists \text { Wt. loss }\end{array}$ & $\begin{array}{l}\downarrow \text { IL- } 1 \beta^{\mathrm{b}} \\
\downarrow \text { IL-6 } \\
\downarrow \text { TNF- } \alpha^{\mathrm{b}} \\
\downarrow \text { TGF- } \beta 1^{\mathrm{b}}\end{array}$ \\
\hline
\end{tabular}




\begin{tabular}{|c|c|c|c|c|c|c|}
\hline $\begin{array}{l}\text { Sanchez- } \\
\text { Fidalgo et al., } \\
2010[13]\end{array}$ & $\begin{array}{l}\text { DSS in female } \\
\text { C57BL/6 mice }\end{array}$ & o.i., Day 30 & $\sim 3$ & 30 days & $\begin{array}{l}\downarrow \text { Histological damage } \\
\downarrow \text { Rectal bleeding } \\
\downarrow \text { Wt. loss } \\
\downarrow \text { a } \\
\downarrow \text { Diarrhea } \\
\downarrow \text { Colon wt./lt. }{ }^{\mathrm{b}}\end{array}$ & $\begin{array}{l}\downarrow \text { TNF- } \alpha^{\mathrm{a}} \\
\downarrow \text { IL-1 } \beta^{\mathrm{a}} \\
\downarrow \text { PGES-1 } \\
\downarrow \text { COX-2 } \\
\text { a } \\
\downarrow \text { iNOS } \\
\downarrow \text { p38 MAPK } \\
\uparrow \text { } \text { IL-10 }^{\text {a }} \\
\text { IL }\end{array}$ \\
\hline $\begin{array}{l}\text { Singh et al., } \\
2010[18]\end{array}$ & $\begin{array}{l}\text { IL-10 deficient } \\
\text { female mice }\end{array}$ & $\begin{array}{l}\text { o.i., for first } 7 \\
\text { days }\end{array}$ & $100^{*}$ & 14 days & $\begin{array}{l}\downarrow \text { Histological score } \\
\uparrow \text { Wt. loss }{ }^{b} \\
\uparrow \text { Colon lt. }\end{array}$ & $\begin{array}{l}\downarrow \text { SAA }^{\mathrm{b}} \\
\downarrow \text { TNF- } \alpha^{\mathrm{b}} \\
\downarrow \text { IL-6 } \\
\downarrow \text { IL-1 } \\
\text { IL }^{\mathrm{b}} \\
\downarrow \text { IFN- } \gamma^{\mathrm{b}} \\
\downarrow \text { COX-2 } \\
\downarrow \text { SIRT1 } \\
\downarrow \text { S } \\
\downarrow \text { CD } 4^{\text {b }} \\
\downarrow \text { CD11b+cellsb }\end{array}$ \\
\hline $\begin{array}{l}\text { Singh et al., } \\
2012[33]\end{array}$ & $\begin{array}{l}\text { DSS in male BALB } / \mathrm{c} \\
\text { mice }\end{array}$ & $\begin{array}{l}\text { Naturally } \\
\text { developed } \\
\text { chronic colitis } \\
\text { by day } 126\end{array}$ & $100^{*}$ & 196 days & $\begin{array}{l}\downarrow \text { Wt. loss } \\
\downarrow \text { Histological score }\end{array}$ & 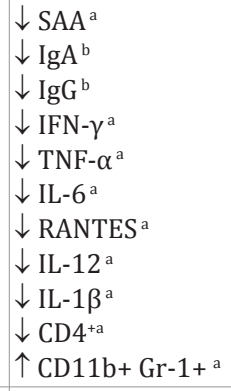 \\
\hline $\begin{array}{l}\text { Yao et al., } \\
2010[20]\end{array}$ & $\begin{array}{l}\text { DSS in male BALB/c } \\
\text { mice }\end{array}$ & o.i., First 7 days & 60 & 14 days & $\begin{array}{l}\downarrow \text { Histological score }{ }^{\text {a }} \\
\downarrow \text { Disease activity index }\end{array}$ & $\begin{array}{l}\downarrow \text { MDA activity } \\
\downarrow \text { MPO activity } \\
\downarrow \text { TNF- } \alpha^{\text {a }} \\
\downarrow \text { IL-8 } \\
\downarrow \text { IFN- } \gamma^{\text {a }} \\
\downarrow \text { p22 } \\
\downarrow \text { phoxa } \\
\downarrow \text { gp91 } 91^{\text {phoxa }} \\
\uparrow \text { SOD } \text { activity }^{\text {a }} \\
\uparrow \text { GSH-Px }\end{array}$ \\
\hline $\begin{array}{l}\text { Yao et al., } \\
2011[15]\end{array}$ & $\begin{array}{l}\text { DSS in male BALB } / \mathrm{c} \\
\text { mice }\end{array}$ & o.i., First 7 days & 35 & 14 days & $\begin{array}{l}\downarrow \text { Disease activity index } \\
\downarrow \text { Histological score }\end{array}$ & $\begin{array}{l}\downarrow \text { MPO activity } \\
\downarrow \text { NF- } \kappa B^{\text {a }} \\
\downarrow \text { TNF- } \alpha^{\text {a }} \\
\downarrow \text { IL-6 }{ }^{\mathrm{a}} \\
\downarrow \text { IL-1 } \beta^{\text {a }}\end{array}$ \\
\hline $\begin{array}{l}\text { Youn et al., } \\
2009[16]\end{array}$ & $\begin{array}{l}\text { DSS in male ICR } \\
\text { mice }\end{array}$ & c.i., 7 days & 10 & 7 days & $\begin{array}{l}\downarrow \text { Wt. loss }{ }^{\mathrm{a}} \\
\downarrow \text { Histological changes } \\
\text { \# Fluid intake } \\
\uparrow \text { Colon lt. }{ }^{\mathrm{b}}\end{array}$ & $\begin{array}{l}\downarrow \text { iNOS }^{\mathrm{b}} \\
\downarrow \text { NF- }^{\circ B} \\
\downarrow \text { STAT3 }^{\text {b }}\end{array}$ \\
\hline \multicolumn{7}{|c|}{$\begin{array}{l}\text { Notes. This table summarizes the results of studies examining the effects of resveratrol in experimental models of IBD, in comparison to groups not } \\
\text { supplementing resveratrol. } \\
\text { a, } p<0.05 ;{ }^{b}, p<0.01 ;{ }^{c}, p<0.001 ; \sim \text {, average ad lib dosage; } \nexists \text { no significant difference; } \\
{ }^{*}, \mathrm{mg} / \mathrm{kg} / \text { every other day.(Doses in } \mathrm{mg} / \mathrm{kg} / \text { day). }\end{array}$} \\
\hline
\end{tabular}

\section{Discussion}

Based on current research, 1 to $100 \mathrm{mg} / \mathrm{kg} /$ day of resveratrol appears to improve clinical symptoms of experimentallyinduced IBD in animals by beneficially modulating inflammatory signaling pathways, oxidative stress, COX-2, acute phase proteins, leukocytes, cytokines, and intestinal microflora. Positive results such as these indicate a potential role for resveratrol in actual cases of IBD.

Anti-inflammatory drugs currently used in the treatment of IBD can induce headache, nausea, pain, diarrhea, sleep disturbance, glucose intolerance, hepatotoxicity, pneumonitis, and tremor [5]. These drugs are used to moderate cytokines, COX-2, and ROS, [5] which, based on current literature, can also be accomplished with resveratrol. In one animal study, $10 \mathrm{mg} /$ $\mathrm{kg} /$ day of resveratrol was equally effective to $300 \mathrm{mg} / \mathrm{kg}$ of sulphasalazine in reducing signs of IBD [21]. Although few studies have directly compared the effects of resveratrol to current medical treatments, resveratrol appears to have characteristics that match such treatments. Studies in both animals with IBD and humans without IBD, have determined no adverse effects of Resveratrol $[10,43,44]$. Its supplementation and effects have not yet been determined for patients with IBD. 
Although experimental induced IBD is a well-established animal model, the reviewed studies are limited by the controlled living conditions and diet, which do not mimic the varying stress factors of free-living conditions that can affect intestinal inflammation [45-47]. Additionally, although many studies contained significance values of findings, they did not report exact values, which hindered quantitative synthesis of data. All studies, however, measured both physical and biological markers of IBD, confirming the results and the true progression of the disease. The strength and significance seen in these studies encourages the continuation of research on resveratrol supplementation on IBD.

Resveratrol appears to improve many attributes of IBD in experimental animal models, suggesting resveratrol as a promising complimentary therapy for IBD. Further research is needed to confirm if such attributes remain effective in humans while refining optimal dosage, discovering pro-drug administration and standardizing measures including instillation of resveratrol, supplementation duration and histological scores.

\section{References}

1. Centers for Disease Control and Prevention. Inflammatory Bowel Disease (IBD). 2011.

2. Kappelman MD, Rifas-Shiman SL, Porter CQ, Ollendorf DA, Sandler RS, Galanko JA, et al. Direct health care costs of Crohn's disease and ulcerative colitis in US children and adults. Gastroenterology. 2008; 135(6): 1907-13. doi: 10.1053/j.gastro.2008.09.012.

3. Ko JK, Auyeung KK. Inflammatory Bowel Disease: Etiology, Pathogenesis and Current Therapy. Curr Pharm Des. 2014; 20(7): 1082-1096.

4. Mattar MC, Lough D, Pishvaian M J, Charabaty A. Current management of inflammatory bowel disease and colorectal cancer. Gastrointest Cancer Res. 2011; 4(2): 53-61.

5. Carter MJ, Lobo AJ, Travis SP. Guidelines for the management of inflammatory bowel disease in adults. Gut. 2004; 53 Suppl 5: V1-16.

6. Kim DH, Hossain MA, Kim MY, Kim JA, Yoon JH, Suh HS. et al. A novel resveratrol analogue, HS-1793, inhibits hypoxia-induced HIF-1 $\alpha$ and VEGF expression, and migration in human prostate cancer cells. Int J Oncol. 2013; 43(6): 1915-24. doi: 10.3892/ijo.2013.2116.

7. Tomé-Carneiro J, Gonzálvez M, Larrosa M, Yáñez-Gascón MJ, GarcíaAlmagro FJ, Ruiz-Ros JA, et al. One-year consumption of a grape nutraceutical containing resveratrol improves the inflammatory and fibrinolytic status of patients in primary prevention of cardiovascular disease. Am J Cardiol. 2012; 110(3): 356-63. doi: 10.1016/j. amjcard.2012.03.030.

8. Tomé-Carneiro J, Larrosa M, Yáñez-Gascón MJ, Davalos A, Zamorano $\mathrm{JG}$, Gonzelvez M, et al. One-year supplementation with a grape extract containing resveratrol modulates inflammatory-related microRNAs and cytokines expression in peripheral blood mononuclear cells of type 2 diabetes and hypertensive patients with coronary artery disease. Pharmacological Research. 2013; 72: 69-82.

9. Zamora-Ros R, Urpi-Sarda M, Lamuela-Raventós RM, MartínezGonzález MÁ, Salas-Salvadó J, Arós F, et al. High urinary levels of resveratrol metabolites are associated with a reduction in the prevalence of cardiovascular risk factors in high-risk patients. Pharmacol Res. 2012; 65(6): 615-20. doi: 10.1016/j.phrs.2012.03.009.
10. Vang O, Ahmad N, Baile CA, Baur JA, Brown K, Csiszar A, et al. What is new for an old molecule? Systematic review and recommendations on the use of resveratrol PLoS One. 2011; 6(6): e19881. doi: 10.1371/ journal.pone.0019881.

11. Docena G, Rovedatti L, Kruidenier L, Fanning A, Leakey NA, Knowles $\mathrm{CH}$, et al. Down-regulation of p38 mitogen-activated protein kinase activation and proinflammatory cytokine production by mitogenactivated protein kinase inhibitors in inflammatory bowel disease. Clin Exp Immunol. 2010; 162(1): 108-15. doi: 10.1111/j.13652249.2010.04203.x.

12. Hollenbach E, Neumann M, Vieth M, Roessner A, Malfertheiner P, Naumann M. Inhibition of p38 MAP kinase- and RICK/NF-kappaBsignaling suppresses inflammatory bowel disease. FASEB J2004; 18(13): 1550-2.

13. Sánchez-Fidalgo S, Cárdeno A, Villegas I, Talero E, de la Lastra CA. Dietary supplementation of resveratrol attenuates chronic colonic inflammation in mice. Eur J Pharmacol. 2010; 633(1-3): 78-84. doi: 10.1016/j.ejphar.2010.01.025.

14. Morgan MJ, Liu ZG. Crosstalk of reactive oxygen species and NF- $\kappa B$ signaling. Cell Res. 2011; 21(1): 103-15. doi: 10.1038/cr.2010.178.

15. Yao J, Wang JY, Liu L, Zeng WS, Li YX, Xun AY, et al. Polydatin ameliorates DSS-induced colitis in mice through inhibition of nuclear factor-kappaB activation. Planta Med. 2011; 77(5): 421-7. doi: 10.1055/s-0030-1250462.

16. Youn J, Lee JS, Na HK, Kundu JK, Surh YJ. Resveratrol and piceatannol inhibit iNOS expression and NF-kappaB activation in dextran sulfate sodium-induced mouse colitis. Nutr Cancer. 2009; 61(6): 847-54. doi: 10.1080/01635580903285072.

17. Cianciulli A, Calvello R, Cavallo P, Dragone T, Carofiglio V, Panaro MA. Modulation of NF- $\kappa$ B activation by resveratrol in LPS treated human intestinal cells results in downregulation of PGE2 production and COX-2 expression. Toxicol In Vitro. 2012; 26(7): 1122-8. doi: 10.1016/j.tiv.2012.06.015.

18. Singh UP, Singh NP, Singh B, Hofseth LJ, Price RL, Nagarkatti M, et al. Resveratrol (trans-3,5,4'-trihydroxystilbene) induces silent mating type information regulation- 1 and down-regulates nuclear transcription factor-kappaB activation to abrogate dextran sulfate sodium-induced colitis. J Pharmacol Exp Ther. 2010; 332(3): 829-39. doi: $10.1124 /$ jpet.109.160838.

19. Sharma M, Mohapatra J, Wagh A, Patel HM, Pandey D, Kadam S, et al. Involvement of TACE in colon inflammation: A novel mechanism of regulation via SIRT-1 activation. Cytokine. 2014; 66(1): 30-9. doi: 10.1016/j.cyto.2013.12.010.

20. Yao J, Wang JY, Liu L, Li YX, Xun AY, Zeng WS, et al. Anti-oxidant Effects of Resveratrol on Mice with DSS-induced Ulcerative Colitis. Arch Med Res. 2010; 41(4): 288-94. doi: 10.1016/j.arcmed.2010.05.002.

21. Abdallah DM, Ismael NR. Resveratrol abrogates adhesion molecules and protects against TNBS-induced ulcerative colitis in rats. Can J Physiol Pharmacol. 2011; 89(11): 811-8. doi: 10.1139/Y11-080.

22. Abdin AA. Targeting sphingosine kinase 1 (SphK1) and apoptosis by colon-specific delivery formula of resveratrol in treatment of experimental ulcerative colitis in rats. Eur J Pharmacol. 2013; 718(13): 145-53. doi: 10.1016/j.ejphar.2013.08.040.

23. Bereswill S, Muñoz M, Fischer A, Plickert R, Haag LM, Otto B, et al. AntiInflammatory Effects of Resveratrol, Curcumin and Simvastatin in Acute Small Intestinal Inflammation. PLoS One. 2010; 5(12): e15099. doi: 10.1371/journal.pone.0015099. 
24. Martín AR, Villegas I, La Casa C, de la Lastra CA. Resveratrol, a polyphenol found in grapes, suppresses oxidative damage and stimulates apoptosis during early colonic inflammation in rats. Biochem Pharmacol. 2004; 67(7): 1399-410.

25. Martín AR, Villegas I, Sánchez-Hidalgo M, de la Lastra CA. The effects of resveratrol, a phytoalexin derived from red wines, on chronic inflammation induced in an experimentally induced colitis model. Br J Pharmacol. 2006; 147(8): 873-85.

26. Avdagić N, Zaćiragić A, Babić N, Hukić M, Seremet M, Lepara O, et al. Nitric oxide as a potential biomarker in inflammatory bowel disease. Bosnian J Basic Med Sci. 2013; 13(1): 5-9.

27. Kolios G, Valatas V, Ward SG. Nitric oxide in inflammatory bowel disease: a universal messenger in an unsolved puzzle. Immunology. 2004; 113(4): 427-37.

28. Cui X, Jin Y, Hofseth AB, Pena E, Habiger J, Chumanevich A, et al. Resveratrol suppresses colitis and colon cancer associated with colitis. Cancer Prev Res (Phila). 2010; 3(4): 549-59. doi: 10.1158/1940-6207. CAPR-09-0117.

29. Chan MM, Mattiacci JA, Hwang HS, Shah A, Fong D. Synergy between ethanol and grape polyphenols, quercetin, and resveratrol, in the inhibition of the inducible nitric oxide synthase pathway. Biochem Pharmacol. 2000; 60(10): 1539-48.

30. Larrosa M, Yañéz-Gascón MJ, Selma MV, González-Sarrías A, Tot S, Cerón JJ, et al. Effect of a low dose of dietary resveratrol on colon microbiota, inflammation and tissue damage in a DSS-induced colitis rat model. J Agric Food Chem. 2009; 57(6): 2211-20. doi: 10.1021/ jf803638d.

31. Martinez J, Moreno JJ. Effect of resveratrol, a natural polyphenolic compound, on reactive oxygen species and prostaglandin production. Biochem Pharmacol. 2000; 59(7): 865-70.

32.Wang D, Dubois RN. The role of COX-2 in intestinal inflammation and colorectal cancer. Oncogene. 2010; 29(6): 781-8. doi: 10.1038/ onc. 2009.421.

33. Singh UP, Singh NP, Singh B, Hofseth LJ, Taub DD, Price RL, et al. Role of resveratrol-induced CD11b(+) Gr-1(+) myeloid derived suppressor cells (MDSCs) in the reduction of CXCR3(+) T cells and amelioration of chronic colitis in IL-10(-/-) mice. Brain Behav Immun. 2012; 26(1): 72-82. doi: 10.1016/j.bbi.2011.07.236.

34. Agarwal S, Smereka P, Harpaz N, Cunningham-Rundles C, Mayer L. Characterization of immunologic defects in patients with common variable immunodeficiency (CVID) with intestinal disease. Inflamm Bowel Dis. 2011; 17(1): 251-9. doi: 10.1002/ibd.21376.

35. Eastaff-Leung, N, Mabarrack N, Barbour A, Cummins A, Barry S. Foxp3+ regulatory $\mathrm{T}$ cells, Th17 effector cells, and cytokine environment in inflammatory bowel disease. J Clin Immunol. 2010; 30(1): 80-9. doi: 10.1007/s10875-009-9345-1.

36. Suri-Payer E, Fritzsching B. Regulatory $\mathrm{T}$ cells in experimental autoimmune disease. Springer Semin Immunopathol. 2006; 28(1): 3-16.

37. Larrosa M, Tomé-Carneiro J, Yáñez-Gascón MJ, Alcántara D, Selma MV, Beltrán D, et al. Preventive Oral Treatment with Resveratrol Proprodrugs Drastically Reduce Colon Inflammation in Rodents. J Med Chem. 2010; 53(20): 7365-76. doi: 10.1021/jm1007006.

38. Rahal K, Schmiedlin-Ren P, Adler J, Dhanani M, Sultani V, Rittershaus $\mathrm{AC}$, et al. Resveratrol has antiinflammatory and antifibrotic effects in the peptidoglycan-polysaccharide rat model of Crohn's disease. Inflamm Bowel Dis. 2012; 18(4): 613-23. doi: 10.1002/ibd.21843.

39. Wung BS, Hsu MC, Wu CC, Hsieh CW. Resveratrol suppresses IL-6induced ICAM-1 gene expression in endothelial cells: effects on the inhibition of STAT3 phosphorylation. Life Sci. 2005; 78(4): 389-97.

40.Zhang, J. Yin and yang interplay of IFN-gamma in inflammation and autoimmune disease. J Clin Invest. 2007; 117(4): 871-873.

41. Larrosa M, González-Sarrías A, Yáñez-Gascón MJ, Selma MV, AzorínOrtuño M, Toti S, et al. Anti-inflammatory properties of a pomegranate extract and its metabolite urolithin-A in a colitis rat model and the effect of colon inflammation on phenolic metabolism. J Nutr Biochem. 2010; 21(8): 717-25. doi: 10.1016/j.jnutbio.2009.04.012.

42. Takaishi H, Matsuki T, Nakazawa A, Takada T, Kado S, Asahara T, et al. Imbalance in intestinal microflora constitution could be involved in the pathogenesis of inflammatory bowel disease. Int J Med Microbiol. 2008; 298(5-6): 463-72.

43. Crowell JA, Korytko PJ, Morrissey RL, Booth TD, Levine BS. Resveratrolassociated renal toxicity. Toxicol Sci. 2004; 82(2): 614-9.

44. Cottart CH, Nivet-Antoine V, Laguillier-Morizot C, Beaudeux JL. Resveratrol bioavailability and toxicity in humans. Mol Nutr Food Re. 2010; 54(1): 7-16. doi: 10.1002/mnfr.200900437.

45. Lee CY. Chronic restraint stress induces intestinal inflammation and alters the expression of hexose and lipid transporters. Clin Exp Pharmacol Physiol. 2013; 40(6): 385-91. doi: 10.1111/14401681.12096.

46. Maric T, Woodside B, Luheshi GN. The effects of dietary saturated fat on basal hypothalamic neuroinflammation in rats. Brain Behav Immun. 2014; 36: 35-45. doi: 10.1016/j.bbi.2013.09.011.

47. Nicholls SJ, Lundman P, Harmer JA, Cutri B, Griffiths KA, Rye KA, et al. Consumption of saturated fat impairs the anti-inflammatory properties of high-density lipoproteins and endothelial function. J Am Coll Cardiol. 2006; 48(4): 715-20. 